\title{
Carnitine Ester Excretion in Pediatric Patients Receiving Parenteral Nutrition
}

\author{
EBERHARD SCHMIDT-SOMMERFELD, DUNA PENN, LORAN L. BIEBER, JANOS KERNER, \\ THOMAS M. ROSSI, AND EMANUEL LEBENTHAL \\ Department of Pediatrics, Section of Gastroenterology, Hepatology and Nutrition, University of Chicago, \\ Chicago, Illinois 60637 [E.S.S., D.P.]; Department of Biochemistry, Michigan State University, East Lansing, \\ Michigan 48824 (L.L.B., J.K.); and Department of Pediatrics, Division of Gastroenterology and Nutrition, State \\ University of New York at Buffalo, Buffalo, New York 14222 /T.M.R., E.L./
}

\begin{abstract}
Carnitine plasma concentrations and the excretion of carnitine and individual carnitine esters were determined in 25 children and adolescents with gastrointestinal diseases receiving carnitine-free parenteral nutrition for at least 1 mo using radiochemical and radioisotopic exchange HPLC methods. Children $<12-y$-old usually had carnitine plasma concentrations $<-2$ SD from the normal mean for age, whereas patients $>12$-y-old had carnitine plasma concentrations within the normal range. Age was the only variable to correlate significantly with plasma carnitine concentrations during parenteral nutrition. Free carnitine (FC) excretion was closely correlated with plasma FC concentrations and minimal at values $<25 \mu \mathrm{mol} / \mathrm{L}$. The excretion of FC and short-chain acylcarnitines was reduced by an order of magnitude in younger compared with older patients and controls, but the excretion of "other" acylcarnitines was less affected. Some of the latter were tentatively identified using gas-liquid chromatographic and mass spectroscopic techniques as unsaturated and/or branched medium-chain carnitine esters with a carbon chain of C8$\mathrm{C10}$. The results suggest that $\mathrm{FC}$ and short-chain acylcarnitine are conserved by the kidney in nutritional carnitine deficiency but that there may be an obligatory renal excretion of other carnitine esters that contributes to the development of hypocarnitinemia in the younger age group. (Pediatr Res 28: 158-165, 1990)
\end{abstract}

\section{Abbreviations}

PN, parenteral nutrition

TPN, total parenteral nutrition

FC, free carnitine

TC, total carnitine

AC, acylcarnitine

SCAC, short-chain acylcarnitine

MCAC, medium-chain acylcarnitine

PEG, polyethylene glycol

IBD, inflammatory bowel disease

Carnitine ( $\beta$-hydroxy- $\gamma$-trimethylamino-butyric acid) plays an important role in energy metabolism by facilitating the transport of FFA across the inner mitochondrial membrane for $\beta$-oxidation

Received June 30, 1989; accepted March 1, 1990.

Correspondence: Eberhard Schmidt-Sommerfeld, M.D. Assistant Professor in Pediatrics. Dept. of Pediatrics, Box 107. Section of Gastroenterology, Hepatology, and Nutrition, University of Chicago, $5825 \mathrm{~S}$. Maryland Avenue, Chicago, IL 60637 .

The MSU-NIH Mass Spectrometry Facility is supported in part by NIH Grant DRR-00480
(1) and regenerating intramitochondrial coenzyme A through transport of acyl-groups out of the mitochondria, thereby maintaining conditions required for oxidative processes (2).

Although carnitine is synthesized de novo in mammalian tissues (3), nutritional carnitine deficiency may occur under certain circumstances if carnitine is not provided with the diet. Infants receiving carnitine-free TPN are particularly prone to develop carnitine deficiency, probably due to impaired carnitine biosynthesis (4) and low carnitine tissue stores (5). Moreover, infants on TPN appear to have a decreased capacity to oxidize fatty acids that can be improved by L-carnitine supplementation $(6,7)$. The question has therefore been raised whether carnitine should be considered an essential nutrient for the infant (8).

It is not known at what time during human development endogenous carnitine biosynthesis becomes adequate. Adults receiving TPN maintain a normal carnitine status over a longer period of time than infants (9). Nonetheless, abnormally low carnitine plasma concentrations were found in $35 \%$ of an adult population on long-term home PN (10). Some of them also had decreased liver carnitine content. The pathogenesis of this is not understood because, in the mature individual, endogenous carnitine biosynthesis should be adequate if precursors are provided with TPN. However, loss of carnitine through the kidney may be an important factor.

FC and/or AC excretion has been shown to be increased during TPN in adult patients with multiple injuries (11), with sepsis, and after surgery (12), but decreased in metabolically stable patients receiving long-term PN (13). Individual carnitine esters were not analyzed in the urine of these patients.

It has been suggested that carnitine plays a role in mitochondrial detoxification of excess or slowly metabolized coenzyme Alinked compounds of endogenous or exogenous origin (14). These acyl moieties are eventually excreted in the urine as AC. This has been well documented in inborn errors of organic acid metabolism. We hypothesized that such a mechanism may be operational even in metabolically normal humans. The associated carnitine loss may lead to decreased carnitine concentrations in susceptible individuals if carnitine is not provided with nutrition.

In the following, we report the carnitine status and excretion of carnitine and carnitine esters in children and adolescents with gastrointestinal diseases requiring $\mathrm{PN}$ for at least 1 mo using a radioisotopic exchange HPLC method recently adapted for human urine (15). In addition, L-carnitine absorption was studied in a child with a functional short bowel.

\section{PATIENTS AND METHODS}

Patients. Twenty-seven children and adolescents with gastrointestinal disease requiring PN for at least 1 mo were enrolled in the study. Informed consent was obtained from the parents and/ 
or patients and the study was approved by the Investigational Review Board of the Children's Hospital of Buffalo. Two children who were later diagnosed as having renal Fanconi syndrome with an abnormally high renal clearance of phosphate and bicarbonate were excluded from the study. The clinical data of the remaining 25 patients are given in Table 1 . They were divided into two groups on the basis of their disease and age. Patients in group $1(n=15)$ were $<12$-y-old and had diseases involving large areas of the small intestine. Patients in group $2(n=10)$ were $>12-y$-old and had IBD affecting the colon and/or the terminal ileum. No patient in group 2 had resection of his/her intestine.

PN was administered through a central venous catheter and consisted of dextrose $(15-25 \%)$, amino acids $(1-3 \mathrm{~g} / \mathrm{kg})$, fat emulsion $(1.5-3.0 \mathrm{~g} / \mathrm{kg})$, electrolytes, minerals, trace elements, vitamins, and heparin (1 IU/mL). Generally, 150-200 nonprotein kcal were given per $g$ of nitrogen. The infusion solutions contained no carnitine. Seventeen patients received their total caloric intake parenterally and eight had variable oral intake during the time of the study (Table 1). About one half of the patients received $\mathrm{PN}$ for more than $3 \mathrm{mo}$ at home and were brought to the hospital for catheter- or disease-related complications. The other half received $\mathrm{PN}$ for $<3$ mo while in the hospital. Three patients (all of them in group 1) had cirrhosis as diagnosed by liver biopsy, which was thought to be associated with their primary disease and/or long-term PN. Five had elevated aminotransferases ( $>3$ times upper normal limit) without any other clinical or biochemical evidence of liver disease. One patient with cirrhosis had cholestasis with conjugated hyperbilirubinemia. Most of the patients with IBD received prednisone during the time of the study. All patients were metabolically stable and in good nutritional status at the time of the study.

Seven patients (mean age 203 mo, range 146-252 mo) with IBD not requiring PN (group 3) were also studied for comparison with group 2 . In addition, eight healthy children (mean age 46 mo, range 2.5-96 mo) were studied as controls for group 1 .

Sample collection. Spontaneously voided urine was collected over a period of $24 \mathrm{~h}(12 \mathrm{~h}$ for children $<3$-y-old $)$. During the collection period, the urine was stored in a refrigerator without preservative. Immediately upon completion of the urine collection, several portions were frozen at $-70^{\circ} \mathrm{C}$ until analysis. During the urine collection, a blood sample was collected and centrifuged, and the plasma frozen at $-70^{\circ} \mathrm{C}$. In 11 patients, a blood sample was collected before the onset of PN and at one or more time points during $\mathrm{PN}$.

Carnitine absorption study. In one patient (no. 7) who had a jejunostomy because of aganglionosis extending from the midjejunum, absorption of L-carnitine in the jejunum was studied after parental consent. After collecting the ostomy output for 1 $\mathrm{h}$ and obtaining a blood sample, $100 \mathrm{mg}$ L-carnitine (Vitacarn, Kendall McGaw, Irvine, CA), 8 g D-xylose, and 1 g PEG 4000 dissolved in $100 \mathrm{~mL}$ water were infused through a nasogastric tube over a period of $5 \mathrm{~min}$. Blood samples were obtained 1 and $4 \mathrm{~h}$ after the infusion, and the ostomy output was collected in hourly increments over a period of $7 \mathrm{~h}$. Carnitine and D-xylose recovered from the ostomy output were related to the recovered nonabsorbable marker PEG 4000 to correct for possible inaccuracies in output collection or spillage. Carnitine and D-xylose absorption were determined by calculating the difference between the amount given and that recovered from the ostomy. In addition, both carnitine and D-xylose were measured in blood.

Carnitine determination. Plasma carnitine was measured by a modification (16) of the radiochemical method of McGarry and Foster (17). The same method was used for urinary carnitine except that urine samples were diluted 1:2 or 1:6 with water. To correct for possible spillage during the collection, the results for urine were expressed as $\mu \mathrm{mol} / \mathrm{g}$ creatinine. Carnitine in ostomy output was determined after precipitation of proteins with an equal volume of $6 \%$ perchloric acid.

Carnitine excretion. Excretion (E) and fractional tubular reabsorption (FTR) of FC were calculated from the concentrations of $\mathrm{FC}$ and creatinine $(\mathrm{Cr})$ in the collected urine $(\mathrm{U})$ and in the plasma sample $(\mathrm{P})$ obtained at midcollection using the following formulas:

$$
\mathrm{E}(\mathrm{nmol} / 100 \mathrm{~mL} \mathrm{GF})=100 \times \frac{\mathrm{U}_{\mathrm{FC}} \times \mathrm{P}_{\mathrm{Cr}}}{\mathrm{U}_{\mathrm{Cr}}}
$$

where GF = glomerular filtrate, and

$$
\operatorname{FTR}(\%)=\left(1-\frac{\mathrm{U}_{\mathrm{FC}} \times \mathrm{P}_{\mathrm{Cr}}}{\mathrm{P}_{\mathrm{Fc}} \times \mathrm{U}_{\mathrm{Cr}}}\right) \times 100
$$

Individual carnitine esters were determined by a modification (15) of the radioisotopic exchange HPLC method originally described by Kerner and Bieber (18). Urine $(0.2 \mathrm{~mL})$ was partially purified and subjected to the radioisotopic exchange reaction in which ${ }^{3} \mathrm{H}$-L-carnitine is enzymatically exchanged into the $\mathrm{AC}$ pool using carnitine acetyltransferase. The labeled carnitine esters were subsequently separated by HPLC, detected by a radioactive flow detector, and the peak areas were integrated by computer.

Peaks of acetyl-, propionyl-, isobutyryl-, and 5-carbon branched-chain $\mathrm{AC}$ were identified by comparing their relative retention times with those of authentic standards (Sigma Chemical Co., St. Louis, MO, or synthesized according to ref. 19). Because urinary MCAC that are branched and/or unsaturated are poor substrates for carnitine acetyltransferase (20), they were indirectly estimated together with other carnitine esters presumably present in the urine but not detectable with the described method by subtracting the concentrations of the known carnitine esters from the total AC (as determined radiochemically). In the following, they are referred to as "other" carnitine esters.

In addition, acetylcarnitine was measured in 15 urine samples by an enzymatic spectrophotometric method $(15,21)$. With this method, acetylcarnitine was unmeasurable in five urine samples with a concentration of $<7 \mathrm{nmol} / \mathrm{mL}$ (as determined by the radioisotopic exchange/HPLC method). There was a strong correlation between the two methods $(r=0.98)$.

Identification of isobutyrylcarnitine and medium-chain carnitine esters. From a patient in group 1 and a control child, $20 \mathrm{~mL}$ urine were lyophylized, dissolved in $2 \mathrm{~mL}$ water, extracted with $20 \mathrm{vol}$ chloroform/methanol $(3: 2, \mathrm{vol} / \mathrm{vol})$ and purified on a 50 $\mathrm{mL}$ column of Bio-Gel P-2. For marker purposes, the isotopic exchange reaction was performed with a small portion of these urine samples containing 1-2 nmol of carnitine. The remaining samples were freeze-dried and added to the labeled portion. The samples were then applied in 12 portions of $0.2 \mathrm{~mL}$ each to the HPLC column and eluted as previously described (18). The compounds in question were repeatedly collected from the column using a fraction collector. The combined fractions were further purified with a Dowex 1 (chloride form) column $(30 \times 5$ $\mathrm{mm}$ ), lyophylized, applied again to the HPLC column and eluted under isocratic conditions with $90 \% 10 \mathrm{mM}$ ammonium formate (pH 7.0) and $10 \%$ methanol. The labeled compounds in question were collected and lyophylized. Small portions were hydrolyzed with $1 \mathrm{~N} \mathrm{KOH}$ and the fatty acid residue analyzed by gas-liquid chromatography (22). The remaining samples were subjected to fast atom bombardment mass spectrometry (22). In one sample, gas-liquid chromatography electron impact mass spectral analysis of the acyl moiety was performed after hydrolysis (22).

Other methods. Urinary creatinine was measured by the picric acid method using a commercially available kit (Sigma Chemical Co.). D-xylose in plasma and ostomy outputs was measured according to Roe and Rice (23), and PEG in ostomy outputs according to Malawer (24).

Statistical evaluation. The Mann-Whitney nonparametric test was used to evaluate differences between patient groups. To test the relationship between FC plasma concentration and urinary excretion, regression analysis was performed after logarithmic transformation of the FC excretion data that provided the best fitting curve. Multiple regression analysis was performed with 
Table 1. Clinical data of 25 children and adolescents receiving $>1$ mo of $P N .{ }^{*}$

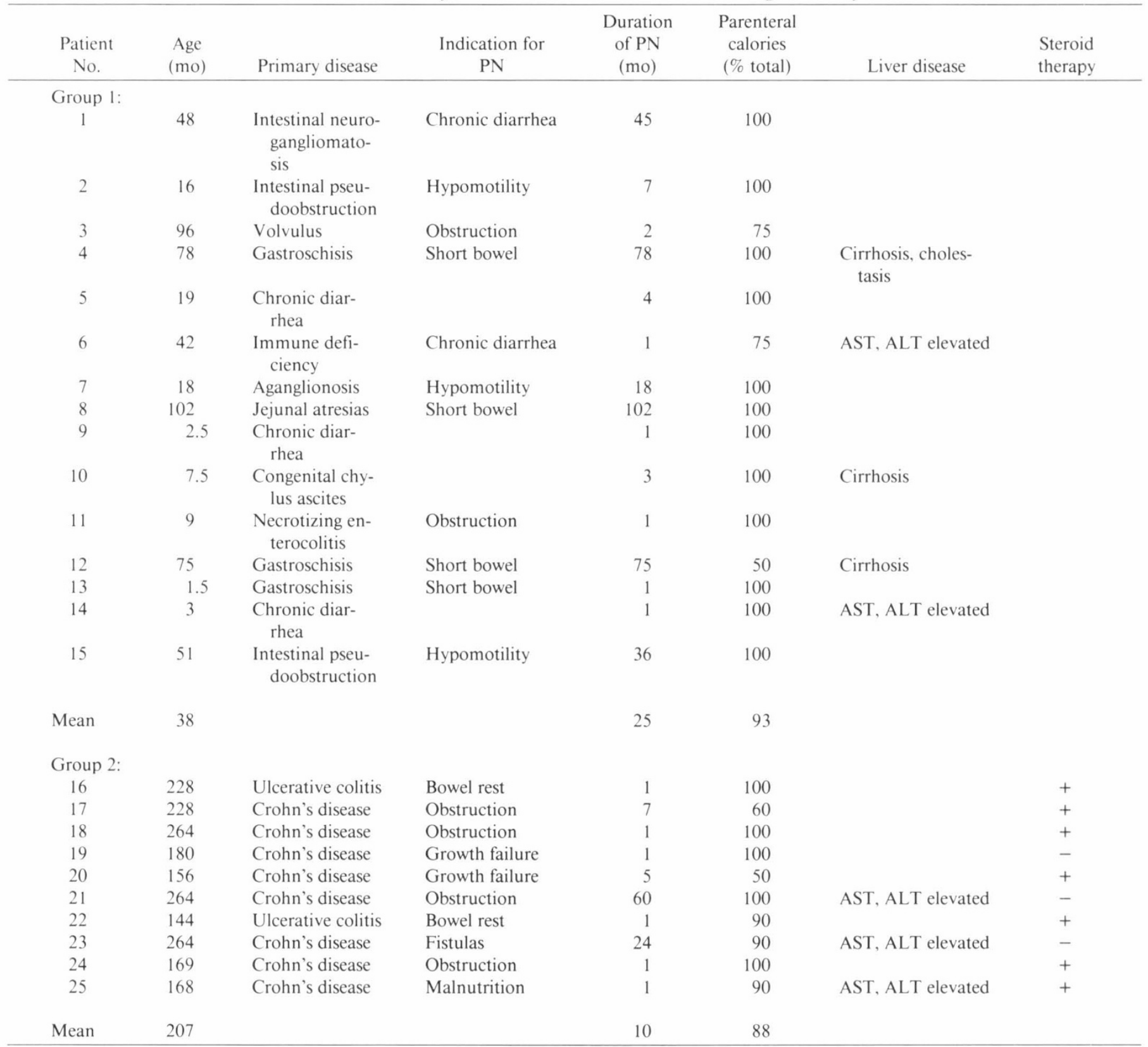

* Patients were divided into two groups on basis of age and type of disease (see text). Aspartate (AST) and alanine (ALT) aminotransferases were elevated $>3$ times upper normal limit where indicated.

plasma TC as the dependent variable and age, duration of $\mathrm{PN}$, and oral intake as the independent variables.

\section{RESULTS}

Plasma carnitine. TC, FC, and AC plasma concentrations were lower in patients in group 1 than in those in group 2 (Table $2)$. Differences in TC and FC plasma concentrations between groups 2 and 3 were much less pronounced. All children except one (no. 11) of group 1 had TC plasma concentrations $<-2$ SD from the normal mean for age (16). With one exception (no. 16), all patients in group 2 had TC and FC plasma concentrations within $2 \mathrm{SD}$ of the normal mean for age.

Among the variables tested by multiple regression analysis, only age was significantly correlated with TC plasma concentrations (expressed as \% of the normal mean, $r=0.68, p<0.001$, Fig. 1). This correlation was still significant even if only patients in group 1 were considered $(r=0.52, p<0.05)$.

In 10 children (four in group 1 and six in group 2), plasma carnitine concentrations could be measured before the onset of PN (Fig. 2). Of the group 1 patients, two (nos. 9 and 14) had decreased and two (nos. 5 and 6) had normal carnitine plasma concentrations at that time. All were malnourished with wt/ht $<3$ rd percentile and hypoalbuminemia. All six patients of group 2 had normal carnitine plasma concentrations before the onset of PN. Four of them were also malnourished. In eight of 10 patients, carnitine plasma concentrations dropped after 1 mo of PN. They remained relatively constant thereafter in those patients who were followed for up to 4 mo (Fig. 2).

Carnitine excretion. Because of exceptionally low carnitine concentrations in plasma and urine, patient no. 16, who had massive blood loss from his colon requiring several transfusions of red blood cells, was excluded from the statistical evaluation of the carnitine excretion data.

Urinary excretion of TC, FC, and AC was lower and the percentage of TC excreted as AC was higher in group 1 compared with group 2 patients or control children (Table 3). Carnitine excretion was not different between patients in groups 2 and 3 . 
Table 2. Plasma concentrations ( $\mu \mathrm{mol} / L)$ of TC, FC, and $A C$ in three groups of patients*

\begin{tabular}{ccccccc}
\hline & $n$ & & $\mathrm{TC}$ & $\mathrm{FC}$ & $\mathrm{AC}$ & $\mathrm{AC} / \mathrm{FC}$ \\
\hline Group I & 15 & $\tilde{\mathrm{x}}$ & $22.3 \dagger$ & $16.6 \dagger$ & $3.8 \ddagger$ & 0.21 \\
& & $\mathrm{R}$ & $8.8-40.4$ & $8.4-34.3$ & $0.4-8.9$ & $0.05-0.54$ \\
& & $\overline{\mathrm{x}}$ & 21.0 & 17.0 & 3.9 & 0.23 \\
Group 2 & 9 & $\tilde{\mathrm{x}}$ & $42.4 \S$ & $33.7 \S$ & 10.4 & 0.31 \\
& & $\mathrm{R}$ & $25.8-61.7$ & $20.9-41.9$ & $2.8-22.4$ & $0.08-0.64$ \\
& & $\overline{\mathrm{x}}$ & 43.4 & 32.3 & 11.4 & 0.35 \\
& & & & & & \\
Group 3 & 7 & $\tilde{\mathrm{x}}$ & 52.3 & 38.0 & 15.8 & 0.43 \\
& & $\mathrm{R}$ & $43.3-75.0$ & $36.1-48.1$ & $3.3-33.0$ & $0.07-0.79$ \\
& & $\overline{\mathrm{x}}$ & 56.1 & 40.5 & 15.5 & 0.39 \\
\hline
\end{tabular}

* Group 1: PN. <12-y-old; group 2: PN, >12-y-old, IBD; group 3: oral nutrition, $>12-y$-old, IBD. $\tilde{x}$, median: $R$, range; $\bar{x}$, mean. Normal range (16): 1 mo-10 y: TC, 28-84; FC, 22-66; AC, 3-32. 10-17 y: TC, 34-77; FC, 22-65; AC, 4-29.

$\dagger p<0.0001$ compared with group 2 .

$\ddagger p<0.002$ compared with group 2 .

$\S p<0.03$ compared with group 3 .

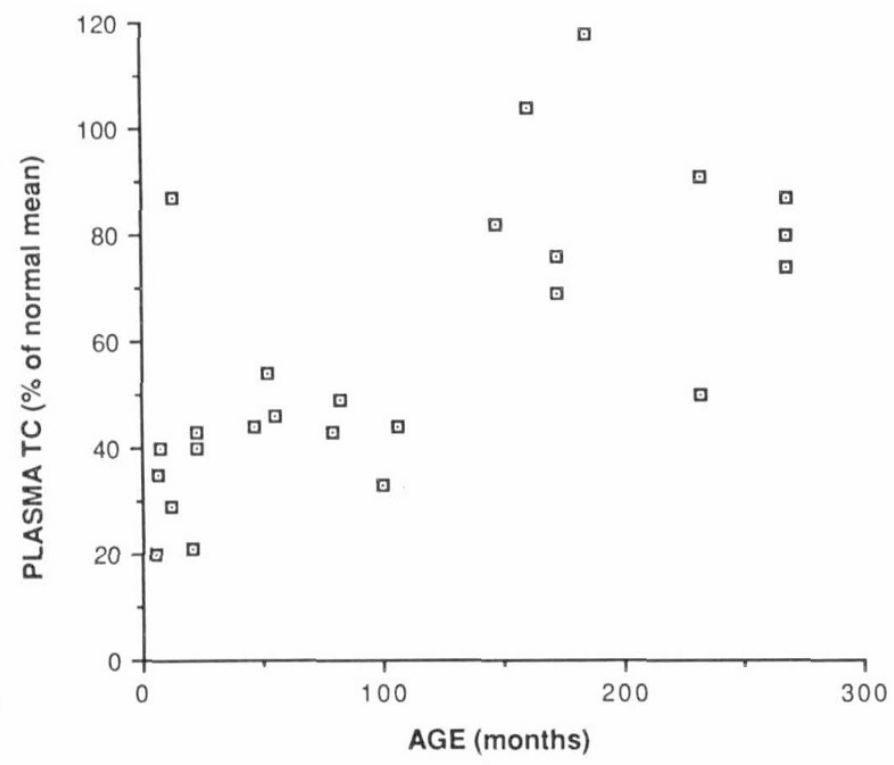

Fig. 1. Relationship between TC plasma concentrations (expressed as $\%$ of the normal mean for age) and age of 25 children and adolescents receiving $\mathrm{PN}$ for $>1$ mo. $\mathrm{y}=36.00 \pm 0.19 \times, r=0.68, p<0.001$.

As shown in Figure 3, there was a positive correlation ( $r=$ $0.80, p<0.001)$ between plasma FC concentrations and FC excretion (expressed as $\mathrm{nmol} / 100 \mathrm{~mL}$ glomerular filtrate). At FC plasma concentrations of $<25 \mu \mathrm{mol} / \mathrm{L}, \mathrm{FC}$ excretion was minimal. The two patients with renal Fanconi syndrome had a high excretion of FC despite low FC plasma concentrations. The mean fractional tubular reabsorption of $\mathrm{FC}$ was $99.7 \%$ in group 1, $96.8 \%$ in group 2 , and $97.1 \%$ in group 3 patients. The difference between groups 1 and 2 was significant $(p<0.015)$, indicating the ability to conserve FC in the former.

The urinary excretion of all SCAC and other AC was lower in group 1 than in group 2 or controls (Tables 4 and 5). However, the differences were more pronounced for SCAC $(\sim 10$-fold $)$ than for other AC (2- to 3-fold). The percentage of TC excreted as SCAC was lower and that excreted as other AC higher in group 1 compared with group 2 or controls (Table 5). Carnitine ester excretion was not different between patients in groups 2 and 3 .

Identity of carnitine esters. Figure 4 shows HPLC chromatograms of urinary carnitine esters of patient no. 11 of group 1 and no. 18 of group 2. In the former (Fig. $4 A$ ), peaks in the area of $\mathrm{MCAC}$ were more pronounced relative to SCAC peaks (C-2 to C-5) than in the latter (Fig. 4B).

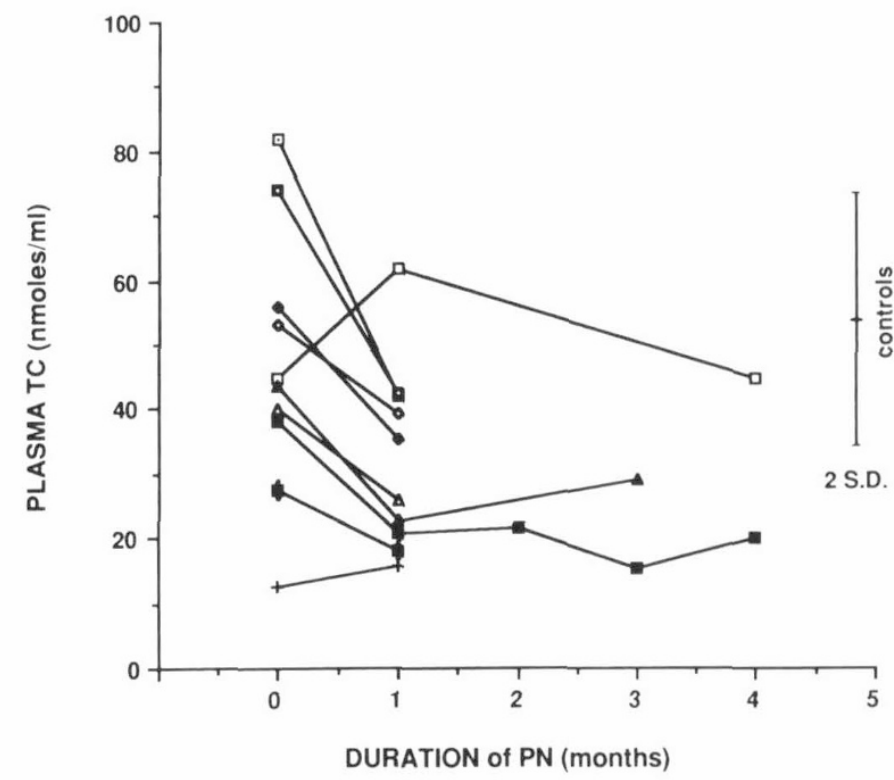

Fig. 2. Plasma TC concentrations of 10 patients before and during PN. Age-matched controls (mean $+2 \mathrm{SD}, n=20$ ) are given for comparison. $\square$, no. $18 ; \mathrm{C}$, no. $22 ;$, no. $25 ;$, no. $24: \square$, no. $19 ; \Delta$, no. $6: \triangle$, no. $16 ;$, no. 5 ; no. $14 ;+$, no. 9 .

Table 3. Urinary excretion ( $\mu$ mol/g creatinine) of TC, FC, $A C$ in three groups of patients and healthy controls (2.5- to 96-mo-old ${ }^{*}$

\begin{tabular}{ccccccc}
\hline & & & & & & $\mathrm{AC} \times 100 /$ \\
& $n$ & & $\mathrm{TC}$ & $\mathrm{FC}$ & $\mathrm{AC}$ & $\mathrm{TC}$ \\
\hline Group 1 & 13 & $\tilde{\mathrm{x}}$ & $81.8+\S$ & $14.4+\S$ & $72.1+\S$ & $83+\S$ \\
& & $\mathrm{R}$ & $43.2-254$ & $4.9-59.6$ & $30.5-195$ & $68-944$ \\
& & $\overline{\mathrm{x}}$ & 105 & 18.3 & 86.9 & 84 \\
Group 2 & 7 & $\tilde{\mathrm{x}}$ & 351 & 170 & 205 & 52 \\
& & $\mathrm{R}$ & $271-755$ & $103-397$ & $154-363$ & $43-68$ \\
& & $\overline{\mathrm{x}}$ & 437 & 212 & 225 & 54 \\
Group 3 & 5 & $\tilde{\mathrm{x}}$ & 317 & 126 & 191 & 64 \\
& & $\mathrm{R}$ & $106-3607$ & $36-1288$ & $70-2319$ & $56-71$ \\
& & $\overline{\mathrm{x}}$ & 940 & 341 & 599 & 63 \\
Controls & 8 & $\tilde{\mathrm{x}}$ & 512 & 272 & 235 & 52 \\
& $\mathrm{R}$ & $198-598$ & $55-333$ & $143-320$ & $41-72$ \\
& & $\overline{\mathrm{x}}$ & 455 & 227 & 229 & 53 \\
\hline
\end{tabular}

* The patient groups are the same as in Table 2. $\tilde{x}$, median; R, range; $\overline{\mathrm{x}}$, mean.

$\dagger p<0.001$ compared with group 2 .

$\ddagger p<0.002$ compared with group 2 .

$\S p<0.001$ compared with controls.

In the urine of patient no. 11 , the prominent peak C-4 (retention time, $20.1 \mathrm{~min}$ ) was identified as isobutyrylcarnitine (Table 6). The MCAC in the urine of patient no. 11 and a control child were (partially) identified as shown in Table 6 and Figures 5 and 6. Due to limited amounts of material, only the structure of the compound with a retention time of $36.8 \mathrm{~min}$ on HPLC could be established as 2-methyloctanoylcarnitine. This peak was found in the urine of $60 \%$ of the control children, in all patients in group 3 , and only occasionally in patients receiving $\mathrm{PN}$ (groups 1 and 2). The peaks with retention times of 33.7 and $35.3 \mathrm{~min}$ on HPLC were found in practically all urine samples studied. No octanoylcarnitine (retention time $36.1 \mathrm{~min}$ ) was present in any specimen.

Carnitine absorption study. Absorption of L-carnitine was studied in patient no. 7, who had a jejunostomy. His TC and FC plasma concentrations were 20.4 and $18.0 \mu \mathrm{mol} / \mathrm{L}$, respectively. 


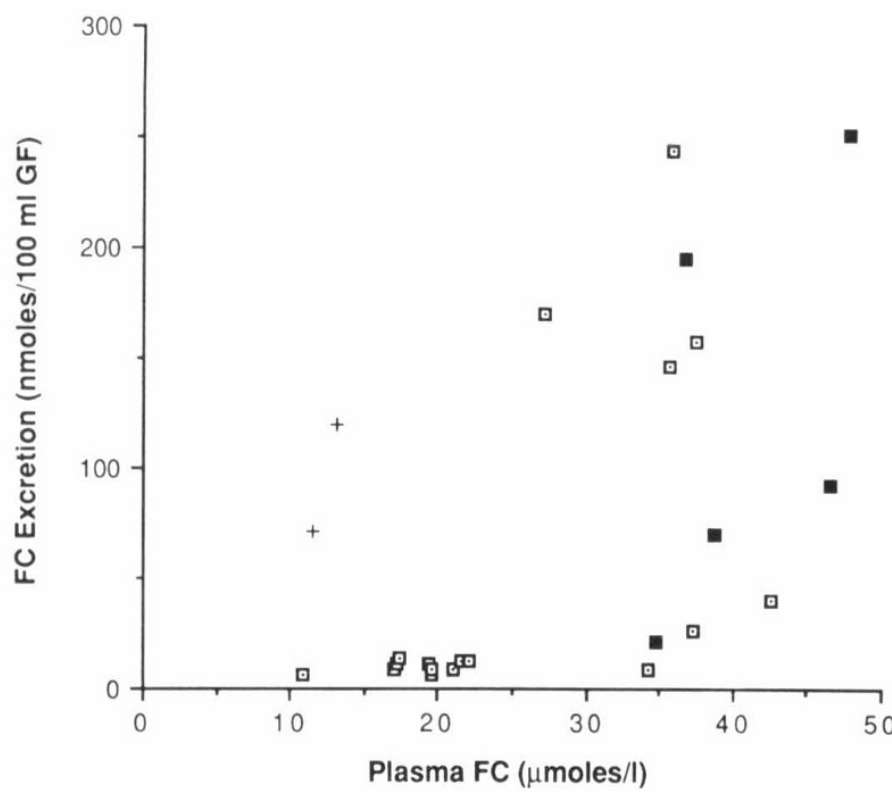

Fig. 3. Relationship between FC plasma concentrations and FC excretion (expressed as $\mathrm{nmol} / 100 \mathrm{~mL}$ glomerular filtrate). $\square$, patients receiving PN (groups $1+2$ ); $\mathbf{a}$, patients with IBD on oral food (group 3 ): +, two patients with renal Fanconi syndrome receiving PN (not included in the statistical evaluation).

Carnitine concentration was minimal $(<2 \mu \mathrm{mol} / \mathrm{L})$ in the initial ostomy output. Four h after L-carnitine and D-xylose administration, all of the administered carnitine was recovered from the ostomy and plasma carnitine concentrations were unchanged. Twelve percent of the administered D-xylose was absorbed and the 1-h D-xylose blood level was $8 \mu \mathrm{g} / \mathrm{dL}$.

\section{DISCUSSION}

In the human, there is indirect evidence that low plasma carnitine concentrations reflect decreased carnitine tissue levels, at least in organs with a relatively high turnover rate for carnitine. Premature infants receiving carnitine-free TPN have decreased carnitine concentrations not only in plasma $(25,26)$, but also in liver, heart, and kidney (5). It has therefore been suggested that infants may have impaired endogenous carnitine biosynthesis. However, it is not known at what age the capacity for carnitine biosynthesis is fully developed in the human. Our finding that age was the only variable to correlate significantly with plasma TC concentrations (expressed as \% of normal mean for age) in our patients receiving $>1 \mathrm{mo}$ of carnitine-free PN suggests that the rate of endogenous carnitine biosynthesis and/or carnitine tissue reserves are insufficient in very young patients to meet carnitine requirements during rapid growth. This is supported by the study of Helms et al. (7), in which young infants (mean age $3 \mathrm{mo}$ ) receiving long-term PN had even lower TC plasma concentrations (mean $9 \mu \mathrm{mol} / \mathrm{L}$ ) than our patients in group 1 .

On the other hand, the type of disease was strikingly different between our two patient groups. Extensive small bowel disease resulting in diarrhea and malabsorption (group 1) may have led to decreased carnitine reserves even before the onset of PN due to impaired intestinal absorption of carnitine or its precursors. This is supported by the finding of abnormally low plasma carnitine concentrations before initiation of $\mathrm{PN}$ in two of the four longitudinally studied patients with malabsorption. Moreover, we recently found carnitine plasma concentrations to be $<-1$ SD from the normal mean for age in four of five children with total villous atrophy due to celiac disease (Hebel E and Schmidt-Sommerfeld E, unpublished data). Carnitine absorption has not been studied in vivo in the human, but it has been shown to be a sodium-dependent active process in biopsy specimens from duodenum and ileum (27). Our absorption study in a child with jejunostomy demonstrates that carnitine absorption can be severely impaired in a patient with a short small intestine.

In two children in group 1, we were able to observe carnitine plasma concentrations over a period of 3-4 mo of PN. In both children, levels dropped after 1 mo to subnormal values and then remained unchanged. This suggests that plasma carnitine concentrations attain a new steady state at a decreased level during carnitine-free nutrition.

Most of our children and adolescents $>12$ y of age with IBD maintained plasma carnitine concentrations in the normal range. Moreover, plasma carnitine concentrations differed only slightly from those in age-matched IBD patients not receiving PN. This indicates that, in this patient population, carnitine reserves and synthetic capacity are probably adequate to maintain a normal carnitine status over an extended period of time on PN (mean $10 \mathrm{mo}$ ). This finding is at variance with a study that reported that $35 \%$ of adult patients with gastrointestinal disease receiving home PN had decreased carnitine plasma concentrations (10). However, these patients were on PN for a longer period of time (mean $32 \mathrm{mo}$ ) and most of them, in contrast to our patients in group 2, had short bowel syndrome.

Carnitine excretion was markedly different between our two

Table 4. Urinary excretion ( $\mu \mathrm{mol} / \mathrm{g}$ creatinine) of short-chain carnitine esters*

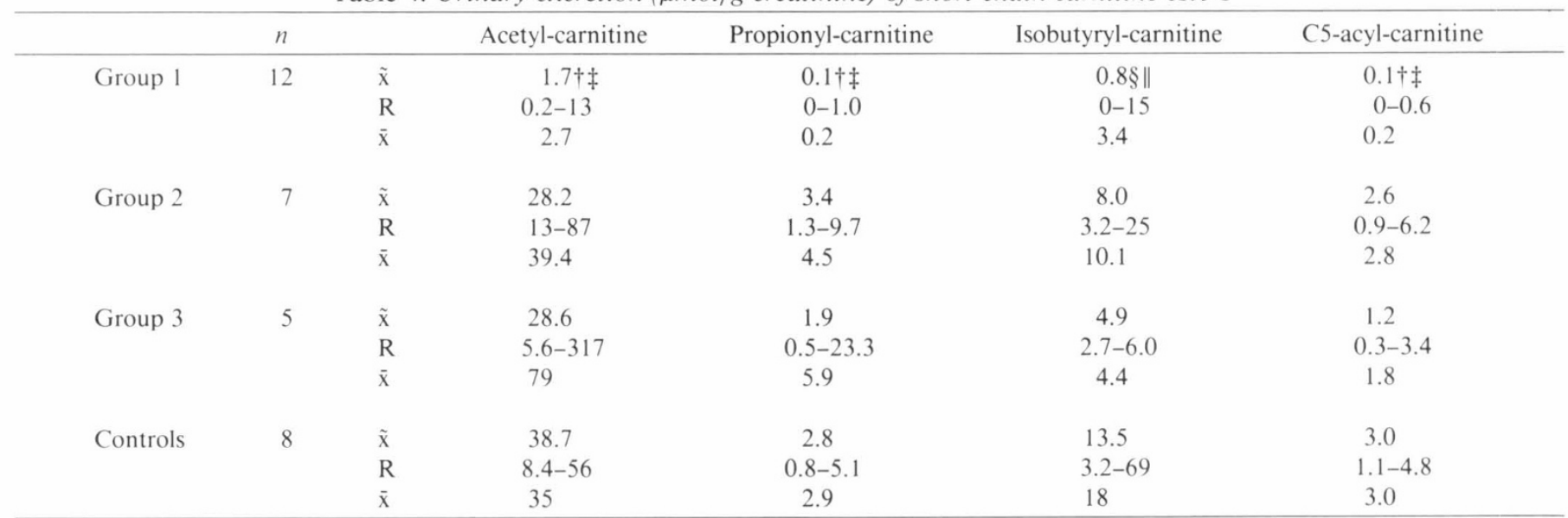

* The groups are the same as in Table 3. $\tilde{\mathrm{x}}$, median; R, range; $\overline{\mathrm{x}}$, mean.

$\dagger p<0.001$ compared with group 2 .

$\ddagger p<0.001$ compared with controls.

$\S p<0.02$ compared with group 2 .

$\| p<0.003$ compared with controls. 


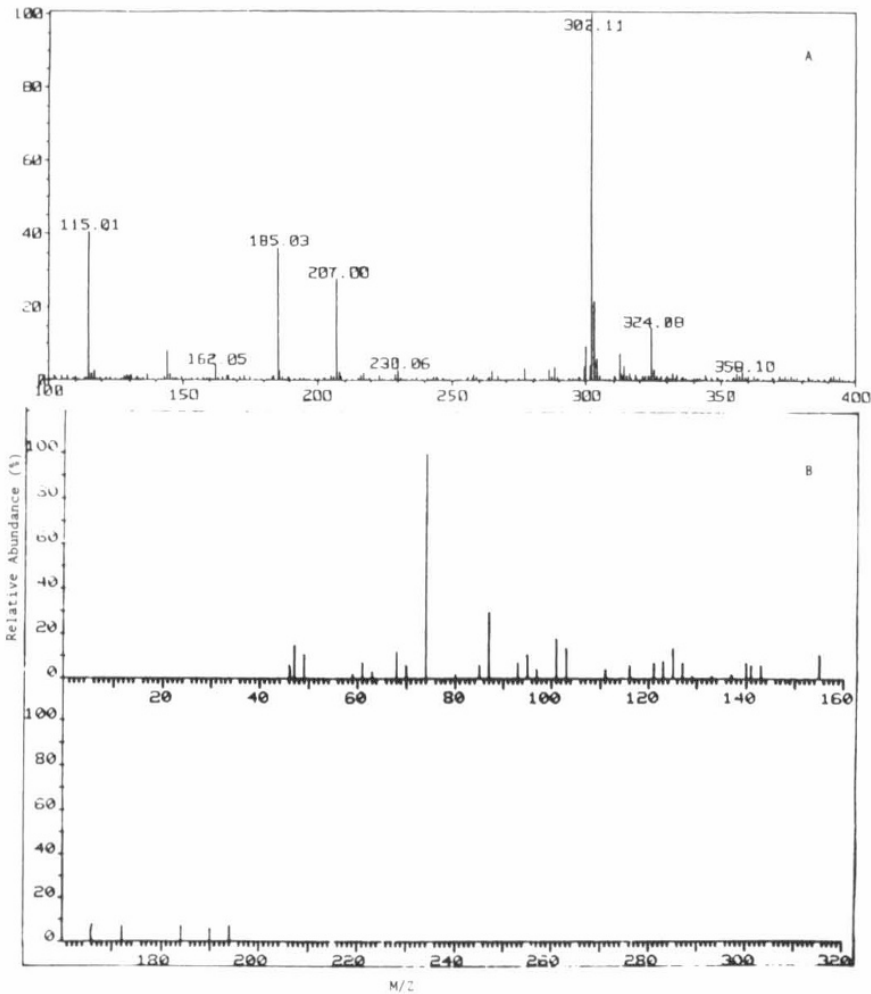

Fig. 6. Identification of MCAC from the urine of a 5-y-old control child. Retention time on HPLC: $36.8 \mathrm{~min}$. A, Fast atom bombardment mass spectral analysis. Protonated $\mathrm{mol} \mathrm{wt}=302($ Na-adduct $\mathrm{mol} \mathrm{wt}=$ 324 ) best fit a 9-carbon saturated $\mathrm{AC}$ (see Table 6). B, Gas-liquid chromatographic electron impact mass spectral analysis of the acyl moiety after hydrolysis: base peak 74 and fragment 87 indicate a methyl branch in position 2 .

apparent renal threshold for FC was $\sim 25 \mu \mathrm{mol} / \mathrm{L}$ in our patient population. This value is lower than the extrapolated value (46 $\mu \mathrm{mol} / \mathrm{L}$ ) reported by Engel et al. (28) in normal adults receiving i.v. L-carnitine. Because all our children with low FC blood concentrations and low urinary FC excretion received $\mathrm{PN}$ and were in the younger age group, we cannot comment as to whether the apparent renal threshold for FC is influenced by $\mathrm{PN}$ or age. Although the kidney was capable of conserving FC when plasma levels were decreased, FC was always detected in the urine. This may reflect secretion of FC by the kidney (29).

Quantitation of individual urinary carnitine esters revealed that the excretion of short (branched)-chain AC was also sharply reduced in our patients in group 1, suggesting that they are reabsorbed by the kidney tubule to a similar extent as FC. This is in agreement with studies by Hokland and Bremer (30) using the perfused rat kidney. However, the excretion of other carnitine metabolites including MCAC was reduced to a much lesser extent in group 1 patients and was appreciable in all patient groups and controls. These AC made up a significantly higher percentage of TC excreted by patients in group 1 compared with group 2 or age-matched controls.

We were able to identify tentatively some of these compounds as unsaturated and/or branched medium-chain carnitine esters with a carbon chain of C-8 to C-10. Kerner et al. (22) have previously identified three medium-chain carnitine esters in urine from adults as 2-methyl- $\Delta^{6}$-octenoyl-, $\Delta^{6}$-octenoyl-, and 2 methyloctanoylcarnitine. The latter two compounds are probably identical to those found in our children.

The origin of these medium-chain carnitine esters, which are distinctly different from octanoyl- and hexanoylcarnitine excreted by patients with medium-chain acyl-CoA dehydrogenase deficiency (31), is unknown. They could be derived from exogenous or endogenous fats. The finding that some of them were excreted in all of our subjects independent of the type of nutrition they received suggests that an endogenous origin is likely. This is supported by our recent finding of complex medium-chain carnitine esters in the urine of newborn infants collected before the onset of feedings (Schmidt-Sommerfeld E, unpublished data). It is also not known in which organs and cell organelles they are formed and whether they are transported through the blood stream. Nor is it known which carnitine acyltransferases are involved in their formation. Some of them may be secreted directly into urine by the kidney. Hokland and Bremer (30) found unidentified carnitine metabolites in rat urine after perfusion of the kidney with labeled carnitine. Judging from their $R_{f}$ values on thin-layer chromatography, these metabolites could have been medium-chain carnitine esters.

It has been suggested that, in renal Fanconi syndrome (32, 33), organic acidurias (34), and primary disturbances of carnitine transport (35), carnitine deficiency results from carnitine loss in the urine. Apart from our two patients with renal Fanconi syndrome, this was not apparent in our hypocarnitinemic patients in group 1. Their kidneys were able to conserve FC and SCAC. However, they lost appreciable amounts of carnitine in the form of MCAC and other yet to be identified carnitine esters with the urine.

On the basis of these findings, one could speculate that carnitine has a role in the removal of presumably slowly metabolized acyl moieties from the body. Such a role has been suggested before for the elimination of fatty acid-like drugs such as valproic and pivaloic acids, which are excreted as their respective carnitine esters $(36,37)$. This normal metabolic pathway proceeds despite hypocarnitinemia and contributes to a state of carnitine deficiency in young children who receive carnitine-free nutrition and in whom rates of endogenous carnitine biosynthesis may be inadequate. It remains to be established whether carnitine supplementation of PN is warranted in this situation.

Acknowledgment. The authors thank the MSU-NIH Mass Spectrometry Facility under the direction of Dr. J. T. Watson, Department of Biochemistry, Michigan State University for their assistance.

\section{REFERENCES}

1. Fritz IB 1959 Action of carnitine on long chain fatty acid oxidation by liver. Am J Physiol 197:297

2. Bieber LL. Emaus R. Valkner K. Farrell S 1982 Possible function of shortchain and medium chain carnitine acyltransferases. Fed Proc 41:2858

3. Englard S 1979 Hydroxylation of $\gamma$-butyrobetaine to carnitine in human and monkey tissues. FEBS Lett 102:297-300

4. Olson AL, Rebouche CJ $1987 \gamma$-butyrobetaine hydroxylase activity is not rate limiting for carnitine biosynthesis in the human infant. J Nutr 117:10241031

5. Penn D, Ludwigs B, Schmidt-Sommerfeld E. Pascu F 1985 Effect of nutrition on tissue carnitine concentrations in infants of different gestational ages. Biol Neonate 47:130-135

6. Schmidt-Sommerfeld E. Penn D. Wolf H 1983 Carnitine deficiency in premature infants receiving total parenteral nutrition. Effect of L-carnitine supplementation. J Pediatr 102(6):931-935

7. Helms RA. Whitington PF, Mauer EC. Catarau EM. Christensen ML, Borum PR 1986 Enhanced lipid utilization in infants receiving oral L-carnitine during long term parenteral nutrition. J Pediatr 109:984-988

8. Borum PR 1985 Role of carnitine during development. Can J Physiol Pharmacol 63:571-576

9. Hahn P, Allardyee DB, Frohlich J 1982 Plasma carnitine levels during total parenteral nutrition of adult surgical patients. Am J Clin Nutr 36:569-572

10. Bowyer BA. Fleming R. Ilstrup D, Nelson J, Reek S. Burns J 1986 Plasma carnitine levels in patients receiving home parenteral nutrition. Am J Clin Nutr 43:85-91

11. Cederblad G, Schildt B, Larsson J, Liljedahl SO 1983 Urinary excretion of carnitine in multiply injured patients on different regimens of total parenteral nutrition. Metabolism 32(4):383-389

12. Nanni G, Pittiruti M, Castagneto M 1983 Carnitine plasma levels during total parenteral nutrition. Am J Clin Nutr 38:339-34l

13. Worthley LIG, Fishlock RC, Snoswell AM 1984 Carnitine balance and effects of intravenous L-carnitine in two patients receiving long-term total parenteral nutrition. J Parenter Enterol Nutr 8:717-719

14. Stumpf DA, Parker D, Angelini C 1985 Carnitine deficiency, organic acidemias and Reye's syndrome. Neurology 35:1041-1045

15. Schmidt-Sommerfeld E. Penn D, Kerner J. Bieber LL 1989 Analysis of 
acylcarnitines in normal human urine with the radioisotopic exchange-high performance liquid chromatography (HPLC) method. Clin Chim Acta 181:231-238

16. Schmidt-Sommerfeld E. Werner D. Penn D 1988 Carnitine plasma concentrations in 353 metabolically healthy children. Eur J Pediatr 147:356-360

17. McGarry JD, Foster DW 1976 An improved and simplified radioisotopic assay for the determination of free and esterified carnitine. J Lipid Res 17:277281

18. Kerner J. Bieber LL 1983 A radioisotopic exchange method for quantitation of short-chain acid-soluble acylcarnitines. Anal Biochem 134:459-466

19. Bohmer T, Bremer J 1986 Propionylcarnitine, physiological variations in vivo. Biochim Biophys Acta 152:559-567

20. Bieber LL, Kerner J 1986 Short chain acylcarnitines: identification and quantitation. Methods Enzymol 123:264-276

21. Pearson DJ. Chase JFA. Tubbs PK 1969 The assay of (-)-carnitine and its Oacyl derivatives. Methods Enzymol 14:612-622

22. Kerner J, Bieber LL 1985 Isolation and identification of methyloctanylcarnitines from human urine. Prep Biochem 15(4):237-257

23. Roe JH, Rice EW 1948 A photometric method for the determination of free pentoses in animal tissues. $\mathrm{J}$ Biol Chem 173:507-512

24. Malawer SJ 1967 An improved turbidimetric analysis of polyethylene glycol utilizing an emulsifier. Gastroenterology 53:250-256

25. Schiff D. Chan G. Seccombe D. Hahn P 1979 Plasma carnitine levels during intravenous feeding of the neonate. J Pediatr 95:1043-1046

26. Penn D, Schmidt-Sommerfeld E, Wolf H 1980 Carnitine deficiency in premature infants receiving total parenteral nutrition. Early Hum Dev 4(1):2324

27. Hamilton JW, Li BUK, Shug AL, Olsen WA 1986 Carnitine transport in human intestinal biopsy specimen. Gastroenterology 91:10-16

28. Engel AG, Rebouche CJ, Wilson DM, Glasgow AM, Romshe CA, Cruse RP
981 Primary systemic carnitine deficiency. II. Renal handling of carnitine. Neurology 31:819-825

29. Rebouche CJ, Engel AG 1980 Significance of renal $\gamma$-butyrobetaine hydroxylase for carnitine biosynthesis in man. J Biol Chem 255:8700-8705

30. Hokland BM, Bremer J 1986 Metabolism and secretion of carnitine and acylcarnitines in the perfused rat kidney. Biochim Biophys Acta 886:223230

31. Schmidt-Sommerfeld E, Penn D, Kerner J, Bieber LL, Rossi TM, Lebenthal E 1989 Quantitation of urinary carnitine esters in a patient with medium chain acyl-CoA dehydrogenase deficiency: effect of metabolic state and Lcarnitine therapy. J Pediatr 115(4):577-582

32. Bernardini J, Rizzo WB, Oalakas M, Bernar J, Gahl WA 1985 Plasma and muscle free carnitine deficiency due to renal Fanconi syndrome. J Clin Invest 75:1124-1130

33. Steinmann B, Bachmann C, Colombo J-P, Gitzelmann R 1987 The renal handling of carnitine in patients with selective tubulopathy and with Fanconi syndrome. Pediatr Res 21:201-204

34. DiDonato S, Rimoldi M, Garavagliz B, Usiel G 1984 Propionylcarnitine secretion in propionic and methylmalonic acidurias, a cause of carnitine deficiency. Clin Chim Acta 139:13-21

35. Treem WR, Stanley CA, Fingold DN, Hale DE, Coates PM 1988 Primary carnitine deficiency due to a failure of carnitine transport in kidney, muscle and fibroblasts. N Engl J Med 319:1331-1336

36. Millington DS, Bohan TP, Roe CR. Yergey AL, Liverato DJ 1985 Valproylcarnitine: a novel drug metabolite identified by fast atom bombardment and thermospray lipid chromatography mass spectrometry. Clin Chim Acta 145:69-76

37. Malegh B, Kerner J, Bieber L 1987 Pivampicillin-promoted excretion of pivaloylcarnitine in human. Biochem Pharmacol 3405-3409

38. Montgomery JA, Mamer OA 1989 Measurement of urinary free and acylcarnitines: quantitative acylcarnitine profiling in normal humans and in severa patients with metabolic errors. Anal Biochem 176:85-95 